\title{
ALCLNE CARATTERISTICHE DEL FRONTE DEL VENTO DURANTE LE TEMPESTE DI SABBIA IN EGITTO
}

\author{
M. G. EL-FANDY
}

In questo lavoro si studiano le regristrazioni di un anemometro di Jine- relative alla direzione ed alla velocita del vento durante di "habools" (") che sorgono nella regione di Kbartoum e le tempeste di sablia che colpiscono le "regioni di Heliopoli e di Almaza (distretlo del Cairo).

Da tale studio si ricava che l'intensiti della tempesta, in particolare la mancanza di visibiliti, dipende non solo dall'aumento della velocita orizzontale del vento ma anche dall"intervallo di tempo the

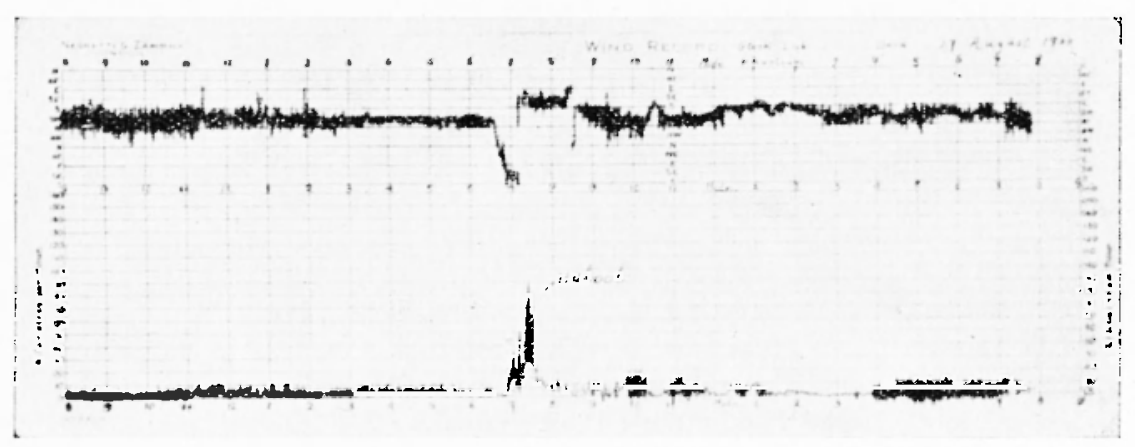

Fig. 1

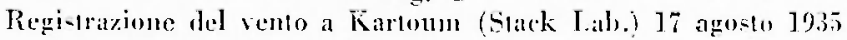

intercorre tra due masimi suceresivi infalti in ogni localita a più lungo periodo corrisponde tempe-ia pia intensa.

Per un colpo di vento singolo la cui durala sia apprezzabile, circa 15 minuti (e it caso normale con habools non slabili) (fig. 1), la risibilità diminuisce rapidamente mentre si innalza una larga nube di polvere. Invece, guando sotto un gradiente di pres-ione crescente la velocita del rento aumenta gradatamente fino ad un valore eritico, si sollerase contemporancamente nubi di sabbia e di polvere. $A_{\text {p- }}$

(1) Fi il nome arabo delle tempere di sablia del Sudan. 
perta lia velocití supera lale limite, mente si susegue una serie di piecoli colpi di rento di breve periodo la risibiliti diminuice notevolmente ma la tempeala ehe si d sviluppata in questo modo b generalmente meno intensa e non si estende verlicalmente al aliezze tanto pronunciats quinto quelle raggiunte nel ratso di tempeste a lipo inslabile.

La fig̣. 1 is la riproduzione di una registrazione all'anemografo di Dine cerguila a Khatroum durante un haboohs ed is chialramente vinibile la registazione e l'andamento del colpo di vento. La fig. 2 i lis riproduzione di una registrazione durante una lempezia sul Cairo ed il rralduale aumcrilo della relocita media è altreltanto chiaramente vi-ibile.

E naturale metlere in relazione l'inlentiat della componente rerticale del vento con l'intentita della tempe-a o-sia con lat densita e Jenemione verlicale della polvere sospesit.

Supponiamo rhe la polvere sia tratertala nell'aria, dopo exere

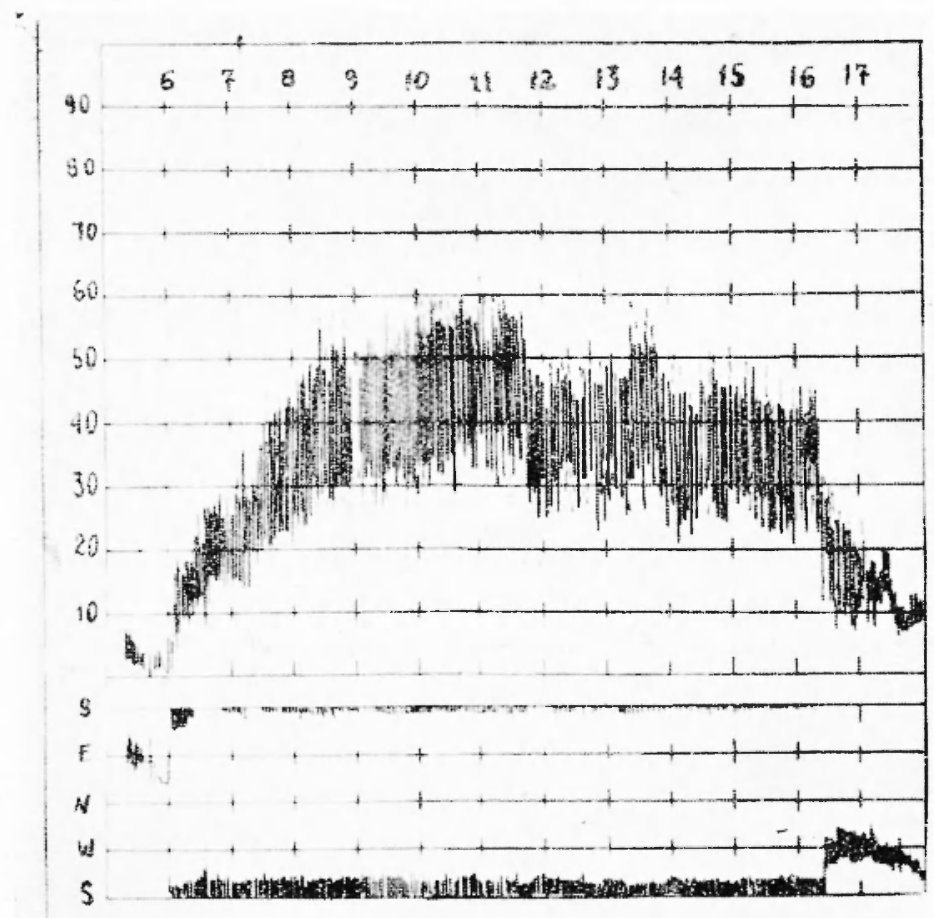

Fig. 2

Regi-trazione del venlo al Cairo del 71 maro 1011. Tempesta di sabbia dalle 0010 alle 1530 (G.MI.'T.). 


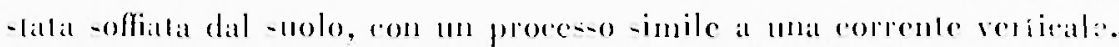

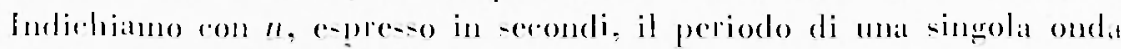
dellat rurva del vento e supponiamo. ele questal posal rappresentarsi medianle una -inu-oide (fig. 3). Si mi-uri il lempo a patrlire dal

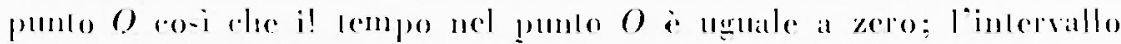

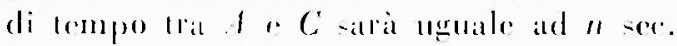

Il rempo retalivo al pumlo t ara allora $-n / 1$ e quello relalivo

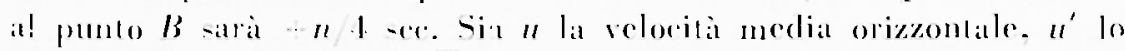

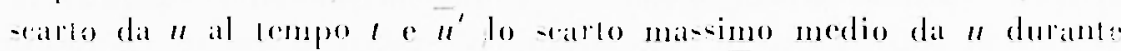

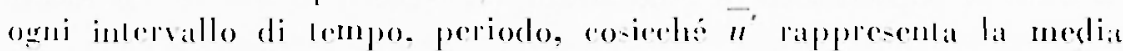

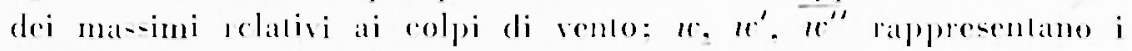
romi-pondenti valori per la emponente verlicale.

fis noto reles quatude à un eolpe di vento si ha un nolevole aumento della velocili del vento -ia in direzione orizontale che in

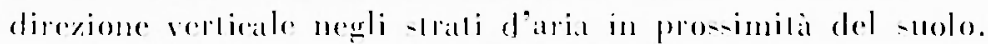

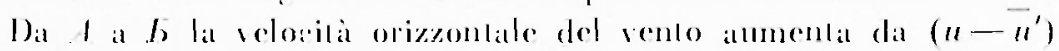

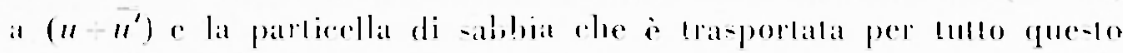

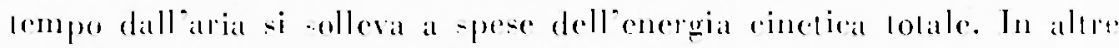

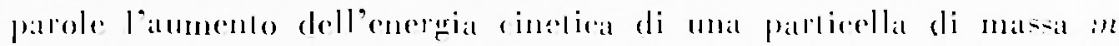

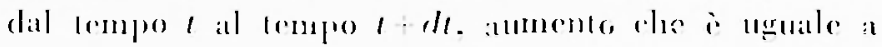

$$
\stackrel{1}{2} m d\left\{\left(u+u^{\prime}\right)^{2}+\left(u+u^{\prime}\right)^{2}\right\}
$$

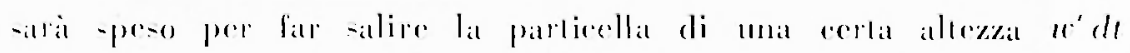
ro-icrhe

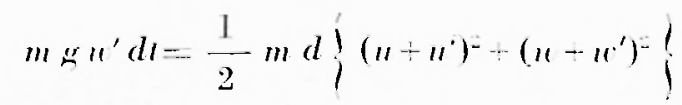

lat rui

$$
u^{\prime}=\frac{1}{2 g} \frac{d}{d t}\left\{\left(u+u^{\prime}\right)^{2}\left(u-w^{\prime}\right)^{\prime}\right\}
$$

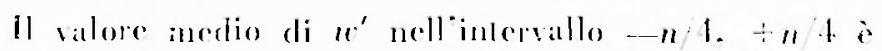

$$
\left.\frac{1}{2} \cdot \sum_{\frac{n}{4}}^{\frac{n}{4}} w^{\prime} d !=\frac{1}{n g} \int_{\frac{n}{4}}^{\frac{n}{4}} d\left(u+n^{\prime}\right)^{2} \cdot\left(u+u^{\prime}\right)\right\}
$$

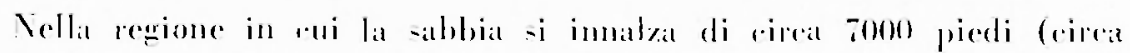

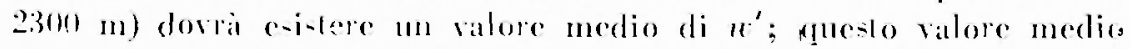
-ia rapperentalo da $Q$ nell"inlervallo con-ideralo, rosicrhe 


$$
\begin{aligned}
& \theta=\frac{1}{g n} ; u^{2}+2 u u^{\prime}+u^{\prime \prime}+u^{2}+2 u u^{\prime}-\left.u^{\prime 2}\right|_{-\frac{n}{4}} ^{\frac{n}{4}} \\
& =\frac{1}{g n}\{1+\bar{u} \cdot+\cdots \bar{u}+ \\
& =\frac{4}{g n} \quad u \bar{u} \cdot u \bar{u}
\end{aligned}
$$

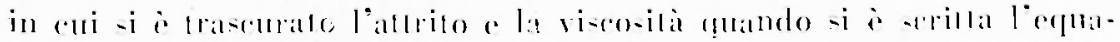
zione della rom-ervizions:

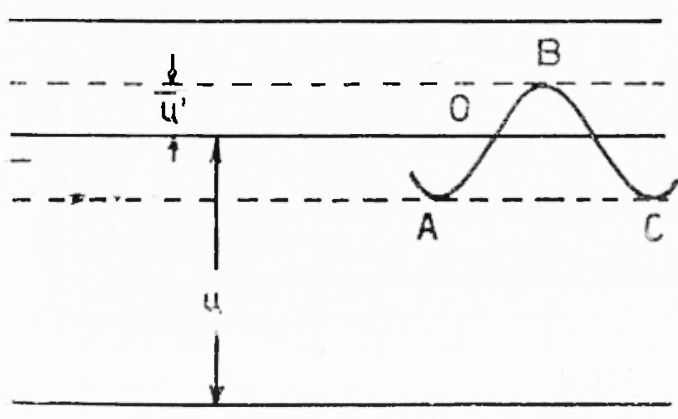

Fig. 3 dellemergial. Quamdo -i pre-enta un -olo rolpo di vento

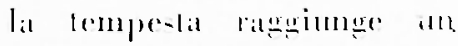
ma-imo nei primi $n \geq$ secondi mentre arrengono nolesoli foostamenti di mame d"allia in dierzione rerliable - la - lla inlemsila dalla relorita dipende dalla veloneila melleal:

olliene

Dallexplazione $: 2 j-i$

$$
\|=\frac{g n}{1 w^{\prime}}-\frac{\| u^{\prime}}{w^{\prime}}
$$

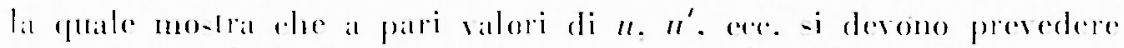
ron wrandi valori di $u$, Erandi velonili verlicali.

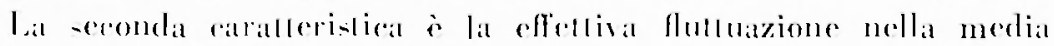
delle velorila e della visibilila durante le lempese di sablial. Tali

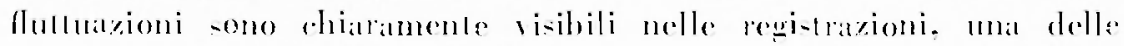

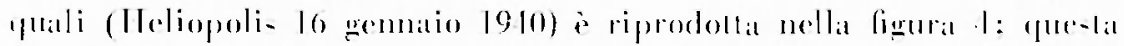
mo-tra la onde steecsive nell’inlervallo di lempo tra le 0900 e lo $2.300)($ lempor lociale).

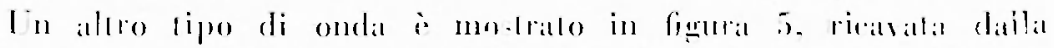
regi-trazone dellamemomelro di Dine di Almaza. di-lrello del Cabro.

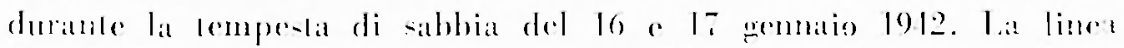

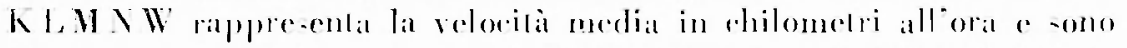

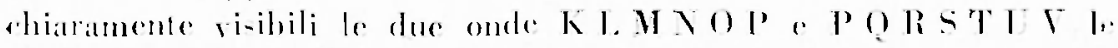
yuali hamo fo sero andamento. Il diario giomaliero ehe i rom-erva 


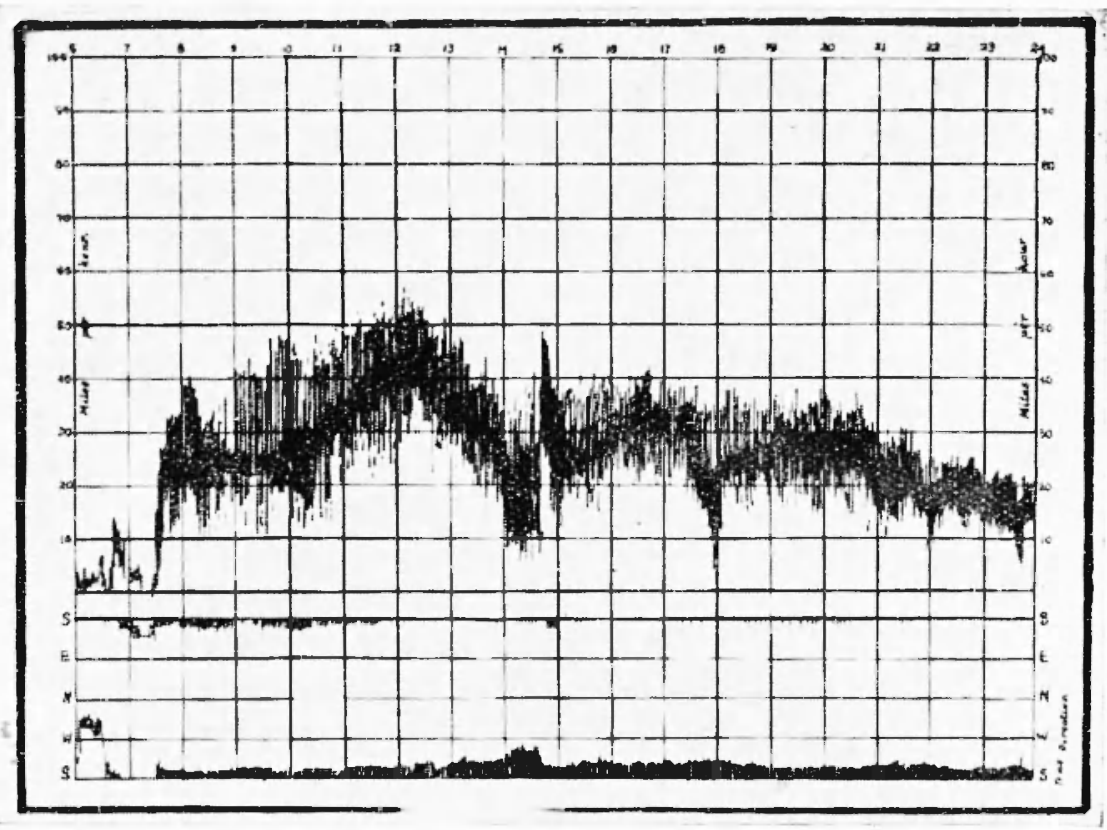

Tig. 4

Regi-1razione dell anomometro di Dina (Ileliopolis 16 genniao 1910 ,

ad Almaza derorive le due tempeste di sibbia dhe si sono manifeslate nell'intervallo di tempo in cui la velorilit del vento aumentil.

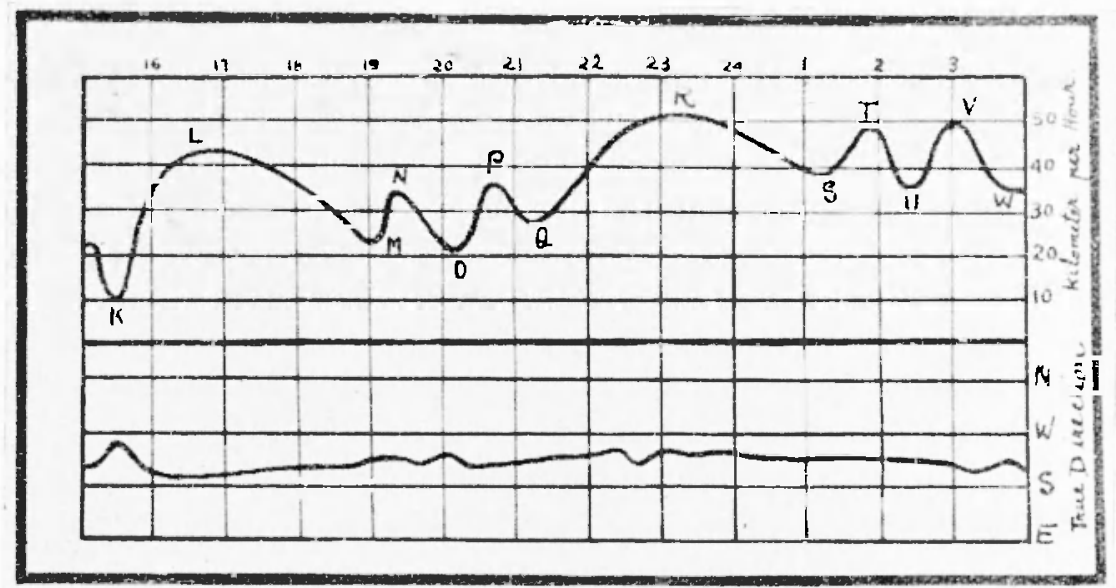

Fig. 5

Registrazione della velociti media e della direzione del vento al Almazal nei giomi 16 e 17 gennaio 1912 
Questa analisi ha un grande valore pratico nel deserto africano poiché è posibile prevedere con un ecrto anticipo l'andamento della curva della velocità media e quando un terzo, o poco più, della curva è slato registrato e raccordato è poscibile determinare l'ora in cui la cempesta arrà fine seguendo il grafico della registrazione dell'inlensili del rento. Tempeste di questo tipo sono frequenti in vicinanza della costa del Nord Africa in primavera ed in inverno e la previ. sione dellora in cui una tempesta ha fine è di particolare interesse sia per lariazione che per il pubblico.

Alexandria, Physics Department, Facully of Science, Faroul $l^{\circ}$ Uniersily, September 19-8.

\section{$R I A S S U N T O$}

Nella presente nota iengono studidte ed analizzale due caralteristiche che presenta l'andamento della velocilà del vento durante le tempeste di sabbia dell Arica del Nord. La prima è che la scarsa visibilità dovuta alla notcvole quantità di sabbia e di polvere sollevate si ha quando compare nella registrazione un caralteristico picco corrispontente al "colpo di vento", in cui ambeduc le componenti, orizzontale e verticale della velocità del vento raggiungono un massimo per circa dieci minuli. I colpi di vento sono associati col fronte fredtlo o con dense nurole tempestose. Le tempeste di sabbia non possono scilupparsi finché una velocitì limite non sia raggiunta. Di tal fatto viene dalt una spicgasione tecrica.

La seconda caratleristica ossertala è la periodicità di tipo ondulatorio dell"andamento della velocitù del vento raggiumla durante le $1 \mathrm{empeste}$ di sabbia. I grafici oltenuli possono generalmente essere ronfrontati con un onda di periodo misurabile ed in tal morlo si può predire la fine della tempesta. 